\title{
PRODUCCIÓN DE ALMÁCIGOS DE CEBOLLA (Allium cepa) BAJO COBERTURA PLÁSTICA EN CAÑAS, GUANACASTE
}

\author{
Roberto Ramírez Matarrita ${ }^{1}$, Jhonny Aguilar Rodríguez², Luis Meza Rodríguez ${ }^{2}$
}

\begin{abstract}
RESUMEN
El estudio se efectuó en San Miguel de Cañas, Guanacaste, en el periodo comprendido entre octubre del 2006 y marzo del 2007. Se evaluaron dos diseños de túnel denominados $1 \mathrm{M}$ y $2 \mathrm{M}$ según su altura a la cresta, (con la finalidad de proteger los almácigos de cebolla del daño mecánico ocasionado por la lluvia) y un testigo a campo abierto. Las variables ambientales registradas mostraron que la temperatura del medio en los días soleados provoca dentro del túnel $1 \mathrm{M}$ un salto térmico de $3,2^{\circ} \mathrm{C}$ con respecto a la temperatura externa, sin afectar fisiológicamente el cultivo. La humedad relativa se mantuvo similar en el interior de los diferentes túneles en relación con el medio externo, independientemente de la condición del día. La radiación procedente del sol disminuyó en un 50\% en los días nublados en comparación con los días soleados. Los almácigos producidos en los túneles registraron las mayores tasas de sobrevivencia con un $90 \%$ para el tratamiento $1 \mathrm{M}, 68 \%$ para el $2 \mathrm{M}$ en comparación con un $10 \%$ del testigo en un lapso de 45 días. Las plántulas sembradas en el túnel $1 \mathrm{M}$ alcanzaron la mayor altura promedio con $24 \mathrm{~cm}$ y el mayor diámetro promedio de bulbo con $5,3 \mathrm{~mm}$. El tratamiento $2 \mathrm{M}$ únicamente superó al $1 \mathrm{M}$ en el peso promedio por plántula; los valores que se obtuvieron fueron $0,72 \mathrm{~g}$ y $0,63 \mathrm{~g}$ respectivamente. Posteriormente a la cosecha de los almácigos, se estableció una parcela experimental con las plantas provenientes de los tratamientos de los túneles $1 \mathrm{M}, 2 \mathrm{M}$ y el testigo, las cuales se llevaron hasta la etapa de producción comercial. Los rendimientos fueron muy similares cercanos a los $7 \mathrm{~kg} / \mathrm{m}^{2}$ indiferentemente del origen del almácigo.
\end{abstract}

Palabras clave: ambiente protegido, almácigos, eficiencia productiva, cosecha, factores climáticos.

\section{INTRODUCCIÓN}

El cultivo de cebolla (Allium cepa) se ha venido desarrollando en Guanacaste, principalmente

en La Fortuna de Bagaces ubicada a una altitud de 800 msnm, donde se está obteniendo el $12 \%$ de la producción nacional (CNP 2004). También, recientemente se iniciaron nuevas experiencias en zonas con altitudes cercanas al nivel del mar, como en el cantón de Cañas, generando expectativas positivas debido a la alta productividad que se ha obtenido en los últimos dos años.
Este rubro ocupa el segundo lugar en importancia económica mundial dentro de las hortalizas, con una superficie de 2116.797 hectáreas y una producción de 52,4 millones de toneladas, siendo China el mayor productor, seguido de E.U.A e India (FAO 2005).

Históricamente, los mejores precios de comercialización de la cebolla en Costa Rica, ocurren en el primer trimestre del año (enero, febrero y marzo) y a medio año (junio, julio y agosto),

1 Instituto Nacional de Innovación y Transferencia en Tecnología Agropecuaria (INTA), Costa Rica. Departamento de Investigación e Innovación. Área de Horticultura Protegida.

2 Instituto Nacional de Innovación y Transferencia en Tecnología Agropecuaria (INTA), Costa Rica. Departamento de Investigación e Innovación. Área de Riego y Drenaje. 
por el desabastecimiento debido al desfase entre las cosechas veraneras y de inverniz. Esto motiva a los productores de Guanacaste para que fijen como meta de producción la primera ventana de comercialización. La duración de los almácigos en campo para esta región es de alrededor de 45 días, por lo tanto, para alcanzar este propósito, los semilleros se deben iniciar a finales de setiembre o a principios de octubre para trasplantar a mediados de noviembre y cosechar 90 días después, que es el tiempo necesario para el desarrollo y engrosamiento del bulbo del híbrido Yellow Granex en esta zona.

Uno de los principales problemas que enfrentan los agricultores para realizar el trasplante al inicio de la época seca, es la producción de almácigos de calidad en la época lluviosa. Las altas precipitaciones, características de esta estación, provocan pérdidas de más del $50 \%$ de la semilla que se siembra, principalmente por el efecto de la escorrentía y el daño mecánico de las gotas de lluvia, aumentando los costos de producción y reduciendo las áreas de siembra programadas. La escasa investigación e información disponible en fuentes bibliográficas que indiquen el efecto de las coberturas plásticas en el desarrollo de las plántulas de cebolla, ha hecho que esta técnica de producción no se haya generalizado en nuestro país.

Los plásticos utilizados en agricultura presentan la ventaja de ser ligeros, con buenas resistencias mecánicas, inalterables, inócuos, impermeables y transparentes a la luz (Castilla 2005). El film plástico más empleado en nuestro país es el polietileno de larga duración, debido a su buena transmisividad de la radiación solar. Según Tesi (2001) el polietileno con un espesor de $0,18 \mathrm{~mm}$ presenta una transmisividad en el rango PAR para radiación directa del 88 al $90 \%$, para radiación difusa del $86 \%$ y para transmisividad del infrarrojo largo del 63 al $65 \%$.

La radiación, temperatura y composición de la atmósfera son factores que pueden modificarse en el interior de una estructura con cobertura plástica, según la naturaleza y propiedades del material de cerramiento, las condiciones de renovación de aire, la forma y dimensión del mismo; así como las posibilidades de evaporación del suelo (Berninger 1989). Durante los días soleados, la radiación solar en su mayor parte atraviesa la cubierta plástica del túnel y es absorbida por las plantas y el suelo mayoritariamente. Estos se calientan y remiten energía, en longitudes de onda dominantes de unas 10 micras, las cuales son interceptadas por el material de cubierta, produciendo un calentamiento en el interior de la estructura (gradiente térmico) conforme a la Ley Wien y que varía según las condiciones de ventilación y estanqueidad del aire (Castilla 2005).

El crecimiento y desarrollo de las plántulas de cebolla está influenciado por los diferentes factores ambientales (luz, temperatura y humedad del suelo) y agrotécnicos (calidad de la siembra, frecuencia y nivel de riego, trabajos culturales, etc.). Las temperaturas óptimas para el desarrollo del cultivo oscilan entre 10 y $25^{\circ} \mathrm{C}$ y la formación del bulbo está influenciada por la duración de luz durante el día, por lo que la respuesta de la planta al fotoperiodo es una cualidad varietal (FAO 1992).

En condiciones del trópico las variaciones en la longitud del día son pequeñas, por lo que la formación del bulbo está más influenciada por la temperatura a que se encuentra sometida la planta, siempre y cuando se trate de materiales de día corto (FAO 1992). Según Corpeño (2004), las plántulas deben contar con cuatro a seis hojas verdaderas, 15 a $20 \mathrm{~cm}$ de altura y alrededor de $6 \mathrm{~mm}$ de diámetro en el cuello falso como parámetros de crecimiento aceptables para iniciar el trasplante del almácigo.

El objetivo del presente trabajo fue evaluar el efecto de dos alturas de túneles sobre el desarrollo fenológico, rendimiento y calidad en la producción de almácigos de cebolla en la época lluviosa y la importancia de estos en la producción comercial de bulbos.

\section{MATERIALES Y MÉTODOS}

El ensayo se realizó en la finca el Cántaro ubicada en San Miguel de Cañas, Guanacaste, en 
el periodo comprendido entre octubre del 2006 y marzo del 2007. El lugar se encuentra a 80 msnm dentro de la zona de vida Bosque Tropical Seco en las coordenadas Lambert norte 259100 norte y 415700 este.

El trabajo de investigación se dividió en dos etapas. En la primera se desarrolló la fase de almácigos donde se evaluó el efecto de la cobertura plástica en forma de túnel sobre el rendimiento y calidad de las plántulas de cebolla para trasplante. Para este fin se utilizaron tres tratamientos: el primero consistió en un túnel de 2 metros de altura a la cresta por 1,5 metros de ancho. El diseño fue en forma de capilla con aberturas laterales de 1,35 m entre el suelo y el borde del plástico. La estructura se construyó con tubo metálico, arcos de varilla lisa metálica de $3 / 8$ " de diámetro y plástico transparente de $0,15 \mathrm{~mm}$ de espesor, con 2,5 metros de ancho. El diseño se designó $2 \mathrm{M}$.

El segundo tratamiento fue un túnel de un metro de altura a la cresta por 1,5 metros de ancho. El diseño fue en forma de capilla con aberturas laterales de $30 \mathrm{~cm}$ entre el suelo y el borde del plástico. La estructura se construyó con cable acerado y el plástico utilizado midió 2 metros de ancho, con un espesor de 0,15 mm. El diseño se designó $1 \mathrm{M}$.

El tercer tratamiento fue una parcela testigo que no contó con cobertura plástica. Los tratamientos se dispusieron en un diseño de bloques al azar, con tres repeticiones. La unidad experimental fue de $38 \mathrm{~g}$ de semilla por tratamiento (equivalente a 8.246 semillas de cebolla), del híbrido Yellow Granex, sembrados a una densidad de seis a siete semillas por centímetro lineal. Cada bloque contó con un diseño de túnel de 3 metros de largo $x$ 1,5 metros de ancho (1M y $2 \mathrm{M}$ ) y una parcela testigo a campo abierto con igual dimensión. Las parcelas estuvieron constituidas por ocho líneas de siembra por surco, con una separación de $15 \mathrm{~cm}$.

Para determinar las variables se tomó una muestra de plántulas en una área de $50 \mathrm{~cm} x$ $50 \mathrm{~cm}$ y se evaluó en el momento de la cosecha: número, peso $(\mathrm{g})$, diámetro $(\mathrm{mm})$ y altura (cm) de plántulas de cebolla. Además, cada semana del periodo de duración del almácigo y a las 9 a.m., se monitorearon las variables ambientales: temperatura $\left({ }^{\circ} \mathrm{C}\right)$ y humedad relativa $(\%)$ con un higrotermómetro digital Radio Shack y la luminosidad (lux) con un luxómetro EXTECH Instruments en el exterior e interior de los túneles a $0,8 \mathrm{~m}$ de altura del suelo .

La segunda fase del experimento se inició posterior a la cosecha de los almácigos. Se estableció una parcela con las plántulas provenientes de los tres tratamientos y de los tres bloques en forma aleatoria, con la finalidad de analizar el efecto de la protección del túnel en la obtención de los almácigos sobre la producción comercial. El modelo estadístico de los tratamientos estuvo dispuesto en bloques al azar con cuatro repeticiones y se utilizaron parcelas de 4 metros de largo por 1,2 metros de ancho, con ocho líneas de surco por parcela y 320 plantas por tratamiento. La separación de las plantas fue de $15 \mathrm{~cm}$ entre líneas de surco y $10 \mathrm{~cm}$ entre plantas. Para evaluar el ensayo las variables analizadas fueron: rendimiento productivo por unidad de área $\left(\mathrm{kg} / \mathrm{m}^{2}\right)$, clasificando las cebollas con base en el diámetro del bulbo $(\mathrm{cm})$. El sistema de riego utilizado fue fertirriego, con tres líneas de cintas de polietileno por era y una descarga de 1,2 l/h.

\section{RESULTADOS Y DISCUSIÓN}

\section{Evaluación de factores ambientales dentro y fuera del túnel}

\section{Temperatura del aire}

La temperatura interior en el túnel de un metro de altura $(1 \mathrm{M})$, mostró un valor máximo de 37 ${ }^{\circ} \mathrm{C}$, cuando la condición de día fue soleado (S.) con un predominio de la radiación directa, originando un salto térmico de $3,2^{\circ} \mathrm{C}$ respecto a la temperatura ambiental; mientras que el túnel $2 \mathrm{M}$ en la misma condición de día registró $33,2^{\circ} \mathrm{C}$, valor muy cercano al de la temperatura ambiental (Cuadro 1). Como lo indica Castilla (2005), la renovación de aire interior por aire exterior conlleva una disminución de su contenido energético, es decir de su entalpía. El túnel $2 \mathrm{M}$ por contar con dos aberturas laterales 
de 1,35 $\mathrm{m}$ de altura, tuvo un mayor potencial de renovación del aire, lo cual evidentemente le proporcionó más eficiencia para eliminar los excesos de calor originados por los efectos convectivo y radiactivo dentro de la estructura que el túnel $1 \mathrm{M}$ con $0,30 \mathrm{~m}$.

Las temperaturas menores se registraron cuando la condición de día fue nublado (N.), obteniéndose en el interior del tratamiento $1 \mathrm{M}, 25,1$ ${ }^{\circ} \mathrm{C}$, mientras en el túnel $2 \mathrm{M}$ se registró $25,2^{\circ} \mathrm{C}$, contra $25^{\circ} \mathrm{C}$ de la temperatura ambiental. La similitud de las temperaturas en los diferentes tratamientos, podría deberse a que en los días con alto índice de nubosidad, la intensidad de la radiación en sus diversas bandas llega a la tierra y disminuye debido a la dispersión de la luz por las nubes y gases de la atmósfera, por lo cual se da un almacenamiento térmico escaso (Berninger 1989), disminuyendo el gradiente térmico según la Ley de Wien, ya que la mayor parte de la energía es liberada por el cultivo y el suelo mediante longitudes de onda larga del infrarrojo.

Cuadro 1. Temperaturas del aire interior y exterior de los túneles registradas, durante los meses de octubre a noviembre del 2006. San Miguel de Cañas, Guanacaste, Costa Rica.

\begin{tabular}{ccccc}
\hline Medición & $\begin{array}{c}\text { Condición } \\
\text { del día }\end{array}$ & 1M & 2M & Testigo \\
\hline 1 & P.N. & 31,1 & 30,6 & 30,7 \\
2 & S. & 37,0 & 33,2 & 33,8 \\
3 & P.N. & 31,4 & 31,6 & 31,6 \\
4 & N. & 25,1 & 25,2 & 25 \\
5 & P.N. & 30,7 & 32,2 & 33,7 \\
\hline
\end{tabular}

P.N. $=$ Parcialmente nublado $\mathrm{N} .=$ Nublado $\mathrm{S} .=$ Soleado

En los días con condición parcialmente nublada (P.N.), en los tres tratamientos se registraron temperaturas intermedias cercanas a los $31^{\circ} \mathrm{C}$ con pocas variaciones.

La parcialidad de las nubes en la atmósfera, genera una intermitencia en la reflexión y dispersión de la radiación proveniente del sol, modificando cuantitativa y cualitativamente el flujo de energía que llega a la tierra, con lo cual se dan temperaturas mayores que en los días nublados pero menores que en los días soleados.

\section{Humedad relativa}

Como se aprecia en el Cuadro 2, la humedad relativa (H.R.) no tuvo variaciones importantes en los diferentes tratamientos, independientemente de la condición del día. La lectura máxima se registró en la medición número cuatro, con una condición de día nublado (N.), alcanzando el túnel $1 \mathrm{M}$ un valor de $81 \%$, el $2 \mathrm{M} 79 \%$ y el testigo $80 \%$. Cuando la temperatura decrece, la energía térmica es baja y existe una tendencia de la humedad del aire a alcanzar la saturación. La humedad ambiental no interviene directamente en la fotosíntesis, pero su papel es indirecto a través de su influencia en la apertura estomática. El cultivo de la cebolla como la mayoría de los cultivos $\mathrm{C} 3$, se desarrolla de manera óptima en humedades relativas del orden del 60 al $80 \%$. Condiciones superiores pueden influir en el desarrollo de enfermedades si genera condensación de agua en las hojas, en cuyas gotas pueden germinar las esporas de los hongos (Castilla 2005).

Cuadro 2. Humedad relativa del aire interior y exterior de los túneles registrada en los meses de octubre a noviembre del 2006. San Miguel de Cañas, Guanacaste, Costa Rica.

\begin{tabular}{ccccc}
\hline & \multicolumn{4}{c}{ Humedad Relativa (\%) } \\
Medición & $\begin{array}{c}\text { Condición } \\
\text { del día }\end{array}$ & 1M & 2M & Testigo \\
\hline 1 & P.N. & 70 & 68 & 67 \\
2 & S. & 59 & 61 & 58 \\
3 & P.N. & 65 & 59 & 61 \\
4 & N. & 81 & 79 & 80 \\
5 & P.N. & 60 & 58 & 58 \\
\hline
\end{tabular}

P.N.=Parcialmente nublado $\mathrm{N} .=$ Nublado $\mathrm{S} .=$ Soleado

La lectura menor se obtuvo con las mayores temperaturas (días soleados). Las diferencias fueron mínimas entre los tratamientos con valores de alrededor del $60 \%$ de humedad rela- 
tiva (Figura 1). En condiciones adecuadas de suministro hídrico y en ausencia de problemas de salinidad, la fotosíntesis no es afectada por la humedad ambiental baja. En general, el desempeño de los túneles para remover los excesos de humedad fue aceptable, ya que no ocurrieron acumulaciones de vapor de agua en el interior de la estructura con respecto a la higrometría del aire exterior, esto originó una escasa presión de organismos fitopatógenos en el cultivo.

Figura 1 Diferencias de temperaturas y humedades relativas registradas en el

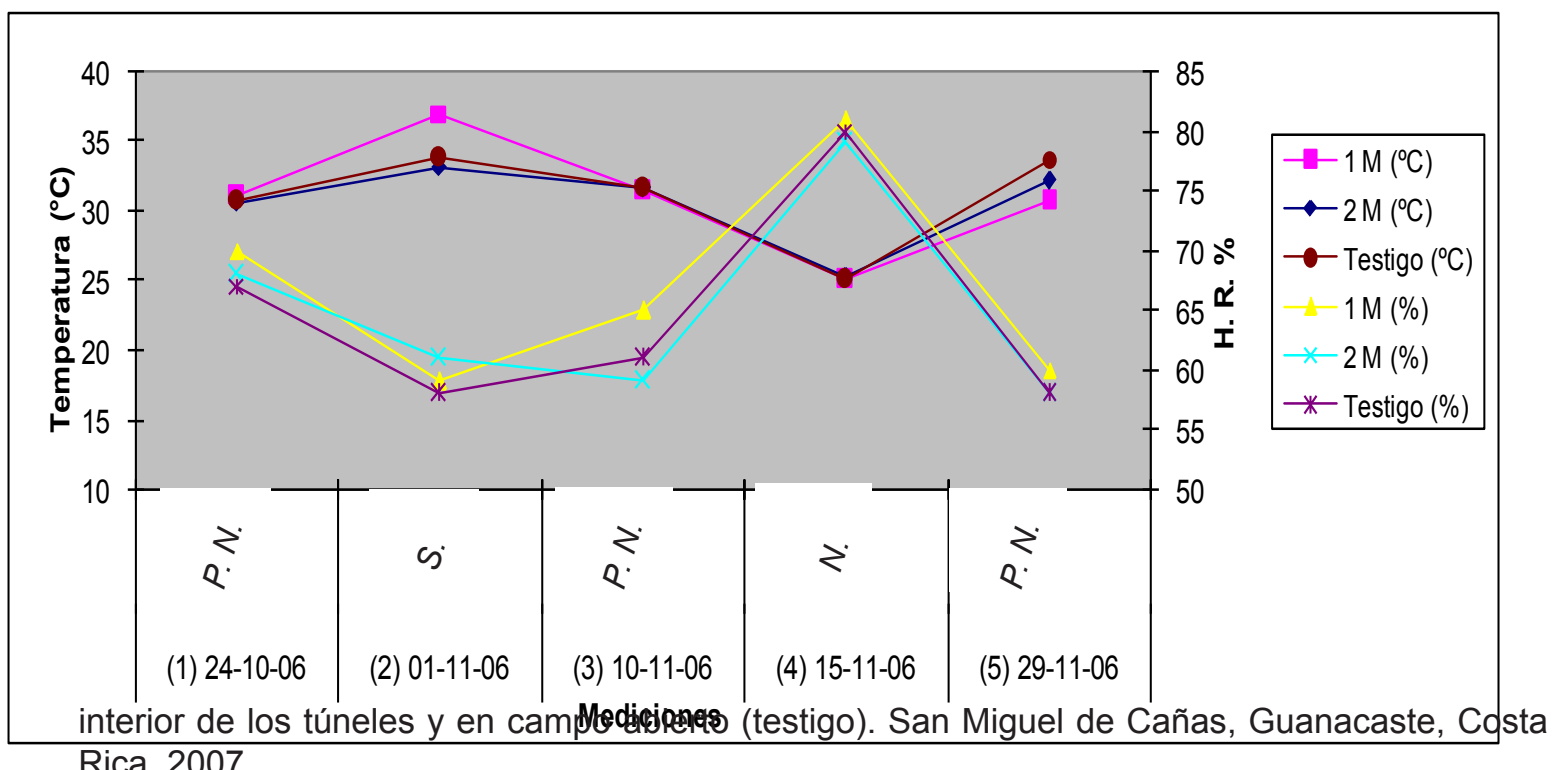

\section{Luminosidad}

El Cuadro 3 muestra los datos de luminosidad en lux. En las mediciones con condición de día

parcialmente nublado (P.N.) la radiación que se registró en el interior de los túneles fue similar a la que se obtuvo en el exterior, sobrepasando siempre los 19.000 lux. Según Langhams et al. (1997) el nivel de radiación adecuado para saturar el sistema fotosintético de muchas plantas tipo C3 como la cebolla, es de alrededor de los $21.600 \mathrm{lux} / \mathrm{m}^{2} / \mathrm{s}$. Esto indica que, las condiciones de radiación donde se efectuó el ensayo (Cañas) son bastante altas, aún en días nublados.

En la época lluviosa prevalece la radiación difusa sobre la directa, la cual procede de todas las direcciones de la bóveda celeste, debido a las reflexiones, desviaciones y dispersiones provocadas por las nubes, gases y aerosoles de la atmósfera (Hanan 1998). Bajo estas circunstancias, la intensidad de la radiación so- bre la superficie plástica disminuye considerablemente, por lo que la transmisibilidad de la cobertura plástica no influye significativamente en el interior de los túneles.

En la medición con una condición de día soleado (S.), donde predomina la radiación directa, el tratamiento $1 \mathrm{M}$ permitió una transmisión de radiación llegando al cultivo 67.300 lux, el tratamiento $2 \mathrm{M}$ registró una radiación de 65.500 lux y en campo abierto de 79.100 lux. Como era de esperar, se presentó una disminución de la transmisibilidad de la luz por efecto del espesor de la cobertura plástica en el interior de los túneles, alcanzando un $15 \%$ en el túnel $1 \mathrm{M}$ y un $17 \%$ en el $2 \mathrm{M}$. Estos datos concuerdan con los citados por Tesi (2001), quien indica que en condiciones de alta luminosidad, la disminución de la transmisividad del polietileno 
de larga duración con espesor de 0,18 mm en la banda PAR oscila entre $10-14 \%$ y para el infrarrojo largo en el orden del $35-37 \%$.
Cuadro 3. Luminosidad (lux) en el interior y exterior de los túneles registrada en los meses de octubre a no-

viembre del 2006. San Miguel de Cañas, Guanacaste, Costa Rica.

\begin{tabular}{ccccc}
\hline Medición & $\begin{array}{c}\text { Condición } \\
\text { del día }\end{array}$ & $\mathbf{1 M}$ & $\mathbf{2 M}$ & Testigo \\
\hline 1 & P.N. & 30.700 & 30.400 & 32.400 \\
2 & S. & 67.300 & 65.500 & 79.100 \\
3 & P.N. & 24.000 & 19.400 & 24.200 \\
4 & P.N. & 50.400 & 41.500 & 53.000 \\
\hline
\end{tabular}

P.N.= Parcialmente nublado $\mathrm{S} .=$ Soleado

Evaluación del desarrollo fenológico, rendimiento y calidad de almácigos de cebolla bajo protección de ambiente

\section{Densidad de plántulas de cebolla por muestra}

El tratamiento de túnel $1 \mathrm{M}$, produjo el mayor número de plantas en la cosecha con un promedio de 618,3 plántulas y una tasa de sobrevivencia del $90 \%$, seguido del tratamiento de túnel $2 \mathrm{M}$ que obtuvo 469 plántulas en la muestra y una tasa de sobrevivencia de 68\% (Cuadro 4). De acuerdo con la prueba de Duncan, no se encontraron diferencias significativas para esta variable. El tratamiento con el menor rendimiento fue el testigo (campo abierto) con 67,3 plántulas y un $10 \%$ de sobrevivencia, presentando diferencias significativas con los tratamientos $1 \mathrm{M}$ y $2 \mathrm{M}$.

Los túneles demostraron ser un medio efectivo para proteger los semilleros del daño mecánico de la lluvia (efecto paragua), aunque en el tratamiento $2 \mathrm{M}$, debido a que contó con una abertura lateral de 1,35 m de altura, se presentaron daños en las líneas de surco de los bordes por alcance de la lluvia, lo que disminuyó el rendimiento con respecto al túnel $1 \mathrm{M}$. En la parcela testigo las mayores pérdidas se dieron antes de la germinación, a causa del salpique de las gotas de lluvia y la escorrentía que expuso la semilla al ambiente.

Cuadro 4. Efecto de los tratamientos sobre la cantidad de plántulas de cebolla cosechadas y tasa de sobrevivencia. San Miguel de Cañas, Guanacaste, Costa Rica. 2007.

\begin{tabular}{cccc}
\hline Tratamiento & $\begin{array}{c}\text { Número de plantas } \\
\text { cosechadas } \\
\left(\mathbf{0 , 2 5} \mathbf{~}^{\mathbf{2}}\right)\end{array}$ & $\begin{array}{c}\text { Número de semillas } \\
\text { sembradas } \\
\left(\mathbf{0 , 2 5} \mathbf{~ m}^{\mathbf{2}}\right)\end{array}$ & $\begin{array}{c}\text { Sobrevivencia } \\
\mathbf{( \% )}\end{array}$ \\
\hline $1 \mathrm{M}$ & $618,3 \mathrm{a}$ & 688 & 90 \\
$2 \mathrm{M}$ & $469 \mathrm{a}$ & 688 & 68 \\
Testigo & $67,3 \mathrm{~b}$ & 688 & 10 \\
\hline
\end{tabular}

Letras distintas indican diferencias significativas $(P \leq 0,05)$. 


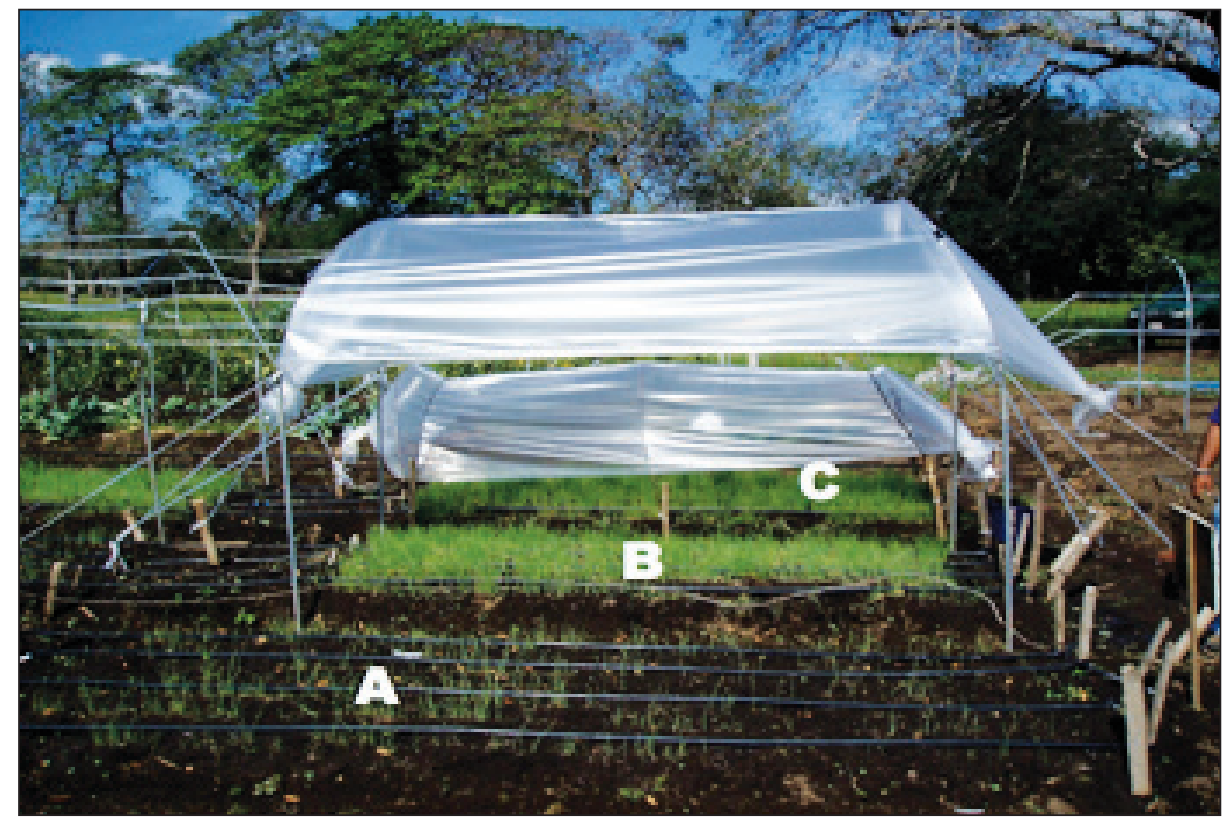

Figura 2. Estado fenológico de los almácigos de cebolla 40 días después de la siembra (A: testigo, B: $2 \mathrm{M}$ y C: 1M).San Miguel de Cañas, Guanacaste, Costa Rica. 2006.

Evaluación de las variables peso, altura de plántulas y diámetro de bulbo de cebolla

Al comparar el peso promedio de las plántulas muestreadas (Cuadro 5), se observa que el tratamiento de túnel $2 \mathrm{M}$ presentó el valor más alto con $0,72 \mathrm{~g}$ por plántula, mientras que en el tratamiento $1 \mathrm{M}$ fue de $0,63 \mathrm{~g}$. Entre estos dos tratamientos no hubo diferencias significativas $(P \leq 0,05)$. El testigo registró el menor peso en la muestra con un valor de 0,29 g por plántula, existiendo diferencias significativas con respecto al tratamiento $2 \mathrm{M}$, pero sin diferir en forma significativa con el tratamiento $1 \mathrm{M}$.

En el tratamiento $1 \mathrm{M}$ la altura promedio fue mayor que en el tratamiento $2 \mathrm{M}$. No hubo diferencias significativas entre estos tratamientos $(P \leq 0,05)$. Mientras tanto el testigo alcanzó una altura promedio de $13,3 \mathrm{~cm}$, quedando muy rezagado en comparación con los tratamientos $1 \mathrm{M}$ y $2 \mathrm{M}$, para los que se detectaron diferencias significativas.

En relación con el diámetro promedio de los bulbos no se observaron diferencias significativas entre los tratamientos $1 \mathrm{M}$ y $2 \mathrm{M}$, registrándose datos promedio de $5,3 \mathrm{~mm}$ para el primero y $5 \mathrm{~mm}$ para el segundo. En el testigo se produjo un pobre desarrollo del bulbo, obteniendo un diámetro de $2,3 \mathrm{~mm}$, muy por debajo de los valores de los tratamientos que se protegieron con cobertura plástica (según la prueba de Duncan $P \leq 0,05)$.

Todos los elementos climáticos afectan de una u otra forma los cultivos, pero algunos autores señalan que la interacción entre la longitud del día y la temperatura influye en el desarrollo de las plantas de cebolla (FAO 1992). Aunque no existieron diferencias notorias en las temperaturas e irradiancia en el interior de los túneles con respecto al medio ambiente por prevalecer la radiación difusa, la disparidad en los promedios de las variables peso, altura y diámetro de bulbo producidos en los tratamientos $1 \mathrm{M}$ y $2 \mathrm{M}$ con respecto al testigo, pudo ocurrir por el hecho de que el suelo de la parcela de control al estar expuesto directamente a la intensidad de las lluvias, sufrió una mayor lixiviación y percolación de elementos nutritivos y lavado de bases; lo que contribuye a la pérdida de la fertilidad y biodiversidad del suelo, repercutiendo en el desarrollo fisiológico y crecimiento de las plantas (Orozco 1999). 
Cuadro 5. Peso, altura y diámetro promedio de plántulas de cebolla obtenidos en la parcela experimental en la cosecha del ensayo. San Miguel de Cañas, Guanacaste, Costa Rica. 2007.

\begin{tabular}{cccc}
\hline $\begin{array}{c}\text { Trata- } \\
\text { miento }\end{array}$ & $\begin{array}{c}\text { Peso de } \\
\text { plantas } \\
(\mathbf{g})\end{array}$ & $\begin{array}{c}\text { Altura de } \\
\text { plantas } \\
(\mathbf{c m})\end{array}$ & $\begin{array}{c}\text { Diámetro } \\
\text { de bulbos } \\
(\mathbf{m m})\end{array}$ \\
\hline $1 \mathrm{M}$ & $0,63 \mathrm{a} \mathrm{b}$ & $24 \mathrm{a}$ & $5,3 \mathrm{a}$ \\
$2 \mathrm{M}$ & $0,72 \mathrm{a}$ & $22,7 \mathrm{a}$ & $5,0 \mathrm{a}$ \\
Testigo & $0,29 \mathrm{~b}$ & $13,3 \mathrm{~b}$ & $2,3 \mathrm{~b}$ \\
\hline
\end{tabular}

Letras distintas indican diferencias significativas $(P \leq 0,05)$.

\section{Rendimiento productivo del cultivo de cebolla en campo}

En lo que se refiere a la productividad por área (Cuadro 6), la mayor producción total por metro cuadrado se obtuvo de las plantas provenientes del túnel $1 \mathrm{M}$; siguiéndole las procedentes del túnel $2 \mathrm{M}$ y por último las del testigo, cuyo rendimiento fue el más bajo. Según la prueba de Duncan no hubo diferencias significativas entre los tratamientos.

Cuadro 6. Rendimiento de las parcelas de cebolla proveniente de los diferentes tratamientos de túneles. San Miguel de Cañas, Guanacaste, Costa Rica. 2007.

\begin{tabular}{cc}
\hline Tratamiento & Rendimiento $\left(\mathbf{k g} / \mathbf{m}^{2}\right)$ \\
\hline $1 \mathrm{M}$ & $7,98 \mathrm{a}$ \\
$2 \mathrm{M}$ & $7,66 \mathrm{a}$ \\
Testigo & $6,81 \mathrm{a}$ \\
\hline
\end{tabular}

Letras distintas indican diferencias significativas $(P \leq 0,05)$.

De acuerdo con Amézquita (1997), una de las ventajas de utilizar la práctica conjunta de riego y fertilización (fertirriego) es la de facilitar la corrección rápida de síntomas carenciales, debido a que los nutrimentos al estar en forma soluble favorecen la asimilación de los mismos por parte de la planta. Esto evidencia que las plán- tulas provenientes de la superficie expuesta al ambiente (testigo), aunque presentaron menor desarrollo vegetativo a la hora del trasplante, lograron expresar su potencial genético, obteniendo una producción muy similar a las alcanzadas en las parcelas cuyos almácigos fueron producidos bajo cobertura plástica.

El Cuadro 7 muestra el número y peso de los bulbos de cebolla seleccionados según el diámetro (calibre) y se aprecia que el testigo alcanzó el mayor número de bulbos con diámetros menores a cinco centímetros (segunda calidad) con 27,25 bulbos y un peso promedio de $37 \mathrm{~g}$, seguido del tratamiento $1 \mathrm{M}$ que obtuvo 26,75 bulbos y un peso promedio de $37 \mathrm{~g}$, sin que existiera diferencias significativas con el testigo $(P \leq 0,05)$. El tratamiento $2 \mathrm{M}$ obtuvo el menor número de bulbos con 22,25 y un promedio en los pesos de $36 \mathrm{~g}$. Según el análisis de varianza existen diferencias significativas para la variable número de bulbos con respecto a los tratamientos $1 \mathrm{M}$ y testigo.

En relación con los bulbos cuyos diámetros van de $5,1 \mathrm{~cm}$ y $10 \mathrm{~cm}$ (primera calidad), el tratamiento con la mayor cantidad fue el $1 \mathrm{M}$, con 107,5 bulbos y un peso total en la muestra de $17,35 \mathrm{~kg}$, promediando $160 \mathrm{~g}$ por planta. En este tratamiento el $90,6 \%$ de la producción predominó en esta categoría, muy similar a los tratamientos $2 \mathrm{M}$ con $90,9 \%$ y el testigo con $89,7 \%$. La evaluación de las variables peso y número bulbos no produjo diferencias significativas entre los tratamientos.

En la categoría de los bulbos de cebolla con diámetros mayores a $10 \mathrm{~cm}$ (jumbo), los tratamientos $1 \mathrm{M}$ y $2 \mathrm{M}$ registraron la mayor cuantía con 2,25 y un peso promedio para el primero de $370 \mathrm{~g}$ y para el segundo de $390 \mathrm{~g}$. En esta categoría se recolectó la menor cantidad de bulbos de toda la muestra representando un $4,3 \%$ para el tratamiento $1 \mathrm{M}, 4,8 \%$ para el $2 \mathrm{M}$ y $4,2 \%$ para el testigo. No se encontraron diferencias significativas entre los tratamientos.

El diámetro del bulbo es muy importante en la comercialización de la cebolla, ya que de esto depende el precio de compra. En la actualidad 
RAMÍREZ et al:: Producción de almácigo de cebolla (Allium cepa) bajo cobertura plástica en Guanacaste

los bulbos de cebolla mejor cotizados son los que presentan diámetros entre 5 a $10 \mathrm{~cm}$. Según la FAO (1992), el híbrido Yellow Granex puede lograr bulbos de mayor diámetro, cuando se desarrolla con condiciones ambientales y agrotécnicas favorables. Como se mencionó anteriormente, las condiciones ambientales predominantes en la zona del estudio sumadas al manejo del riego y nutrición son óptimas para potenciar el desarrollo fenológico del cultivo, de ahí que alrededor del $90 \%$ de la producción que se obtuvo fue de primera calidad.

Cuadro 7. Número y peso de bulbos de cebolla seleccionados por diámetro. San Miguel de Cañas, Guanacaste, Costa Rica. 2007.

\begin{tabular}{|c|c|c|c|c|c|c|}
\hline Tratamiento & $\begin{array}{l}N^{\circ} \text { bulbos } \\
<5 \mathrm{~cm}\end{array}$ & $\begin{array}{c}\text { Peso bulbos } \\
\text { (kg) } \\
<5 \mathrm{~cm}\end{array}$ & $\begin{array}{l}N^{\circ} \text { bulbos } \\
5,1-10 \mathrm{~cm}\end{array}$ & $\begin{array}{c}\text { Peso bulbos } \\
(\mathrm{kg}) \\
5,1-10 \mathrm{~cm}\end{array}$ & $\begin{array}{l}N^{\circ} \text { bulbos } \\
>10 \mathrm{~cm}\end{array}$ & $\begin{array}{c}\text { Peso bulbos } \\
\text { (kg) } \\
>10 \mathrm{~cm}\end{array}$ \\
\hline $1 \mathrm{M}$ & $26,75 a$ & $0,98 \mathrm{a}$ & $107,5 a$ & $17,35 \mathrm{a}$ & $2,25 a$ & $0,83 a$ \\
\hline $2 \mathrm{M}$ & $22,25 b$ & $0,8 a$ & 105,75 a & $16,7 \mathrm{a}$ & $2,25 a$ & $0,88 a$ \\
\hline Testigo & $27,25 \mathrm{a}$ & $1,0 \mathrm{a}$ & $92 \mathrm{a}$ & 14,68 a & $1,75 \mathrm{a}$ & 0,68 a \\
\hline
\end{tabular}

Letras distintas indican diferencias significativas $(P \leq 0,05)$.

\section{CONCLUSIONES}

1. De acuerdo con la época del año (octubrenoviembre) en que se realizó la evaluación de los parámetros ambientales de la investigación, la altura de la abertura lateral de los túneles, no ocasionó fluctuaciones importantes en la temperatura y humedad relativa interna cuando los días fueron parcial o totalmente nublados. En los días soleados, el túnel $1 \mathrm{M}$ no demostró ser tan eficiente en la renovación de aire como el $2 \mathrm{M}$ ya que presentó un salto térmico del orden de los $3,2^{\circ} \mathrm{C}$, sin que esto afectara negativamente el desarrollo fisiológico final de las plántulas de cebolla durante el ciclo de semilleros.

2. El túnel de dos metros de altura a la cresta (2M), comprobó tener una excelente tasa de renovación de aire manteniendo la temperatura y humedad relativa muy similar a la ambiental, pero no fue tan eficiente en la contención del agua de lluvia, como sí ocurrió con el $1 \mathrm{M}$, ya que penetró en las líneas de surco de los bordes, causando lavados en esas áreas.

3. La radiación solar en el interior de los túneles disminuyó en un $15 \%$ en el tratamiento $1 \mathrm{M}$ y un $17 \%$ en el $2 \mathrm{M}$ en los días soleados por efecto del grosor de la cobertura plástica. En los días parcialmente nublados, donde prevalece la radiación difusa, no hubo un efecto perjudicial del plástico en la transmisión de luz. La intensidad lumínica aún en días con alta proporción de nubosidad, fue idónea para alcanzar la tasa de saturación fotosintética, lo que demuestra el potencial agroecológico de la zona para producir almácigos en la época lluviosa, trasplantarlos al inicio del verano y cosechar al final del primer trimestre del año.

4. La protección de las plántulas de cebolla por medio de la cobertura plástica, contra el daño provocado por el salpique de las gotas de lluvia, lixiviación y escorrentía, tiene un efecto positivo sobre la actividad, al aumentar significativamente el porcentaje de sobrevivencia y conseguir una mayor precocidad en el desarrollo fisiológico de las plántulas, aunque esto no es determinante en el rendimiento final en campo.

5. Las plantas procedentes de los almácigos producidos en túneles obtuvieron el mayor rendimiento productivo en campo, pero se comprobó cómo las plantas trasplantadas de la parcela testigo que estuvo expuesto al aire libre, el rendimiento fue muy similar 
al de los tratamientos $1 \mathrm{M}$ y $2 \mathrm{M}$, quedando evidente que el manejo agronómico que se le brinda al cultivo desde el trasplante hasta la cosecha es lo que define finalmente el rendimiento productivo.

\section{RECOMENDACIONES}

De acuerdo con los resultados, el túnel que provee mayor protección contra el efecto de la Iluvia es el que tiene una altura de un metro a la cresta y aberturas laterales de $30 \mathrm{~cm}(1 \mathrm{M})$. Este modelo se puede utilizar de forma permanente durante toda la época lluviosa sin necesidad de estar abriendo y cerrando la cobertura plástica.

Para encontrar una altura idónea que mitigue la penetración de la lluvia y que a la vez no presente un salto térmico considerable que pueda afectar en un periodo prolongado de sequía en la época inverniz, se debe continuar con el proceso de investigación que evalúe diferentes alturas y aberturas laterales para mejorar la eficiencia termodinámica del diseño.

\section{LITERATURA CITADA}

Alpi, A. Y.; Tognonl, F. 1999. Cultivo en invernadero. Madrid, España. Ediciones Mundi-prensa. 347 p.

Amezquita, E. 1997. Fertirrigación: Requerimientos de agua y nutrición de cultivos de flores. Sociedad Colombiana de la Ciencia del Suelo. Bogotá, Colombia. p. 107-126.

Berninger, E. 1989. Cultures florales de serre en zone méditerranéenn française: Eléments climatiques et physiologiques. París. Ed. INRA, PHN - Revue Horticole p. $34-37$.

Castilla, N. 2005. Invernaderos de plástico. Madrid, España. Editorial MundiPrensa. p. 25-232.
CNP (Consejo Nacional de Producción, CR). 2004. Boletín \# 5 de cebolla. (En línea). San José, Costa Rica. Consultado el 22 de abril del 2008. Disponible: http://www.mercanet.cnp. go.cr/SIM/Frutas_y_Vegetales/Históricos/ Cebolla/2004/cebolla.

Corpeño, B. 2004. Manual de campo para la construcción y siembra de semilleros de cebolla. Centro de Inversión, Desarrollo y Exportación de Agronegocios (IDEA). El Salvador. p. 2-12.

FAO (Organización de las Naciones Unidas para la Agricultura y la Alimentación, IT), 1992. Producción, poscosecha, procesamiento, y comercialización de ajo, cebolla y tomate. Editores. Juan Izquierdo, Gaetano Paltrinieri y Ciro Arias. Santiago, Chile. Oficina Regional de la FAO para América Latina y el Caribe. p. $135-171$.

2005. Base de datos FAOSTAT. (En línea). San José, Costa Rica. Consultado el 13 de mayo del 2008. Disponible en http://www.apps.fao.org/ default.htm

Hanan, J. 1998. Greenhouses: Advanced technology for protected cultivation. Boca Raton. Florida. USA. Ed. CRC Press. $684 \mathrm{p}$.

Langhams, R.; Tibbitts, T. 1997. Plant growth chamber handbook. USA. Ed. lowa State Univ. Press $240 \mathrm{p}$.

Orozco, J. 1999. Fertilizantes orgánicos y su aplicación en el cultivo del banano. Memorias del taller internacional Producción de Banano orgánico $y$, o, ambientalmente. EARTH, Guácimo, Costa Rica. p. 82-88.

Tesi, R. 2001. Medios de protección para la hortofruticultura y el viverismo. Traductor: Mateo, J. M. Madrid. Ed. Mundi-Prensa. $43 \mathrm{p}$. 